\section{Pattern of somatic mutations in patients with Waldenström macroglobulinemia or IgM monoclonal gammopathy of undetermined significance}

\author{
Marzia Varettoni, ${ }^{1}$ Silvia Zibellini, ${ }^{1}$ Irene Defrancesco, ${ }^{1}$ Virginia Valeria Ferretti, ${ }^{2}$ \\ Ettore Rizzo, ${ }^{3}$ Luca Malcovati ${ }^{1,2}$, Anna Gallì, ${ }^{1}$ Matteo Giovanni Della Porta, ${ }^{4}$ \\ Emanuela Boveri, ${ }^{5}$ Luca Arcaini, ${ }^{1,2}$ Chiara Candido, ${ }^{1}$ Marco Paulli ${ }^{5}$ and Mario \\ Cazzola ${ }^{1,2}$ \\ ${ }^{1}$ Department of Hematology Oncology, Fondazione IRCCS Policlinico San Matteo, Pavia; \\ ${ }^{2}$ Department of Molecular Medicine, University of Pavia; ${ }^{3}$ en Genome srl, Pavia; ${ }^{4}$ Cancer \\ Center, IRCCS Humanitas Research Hospital \& Humanitas University, Milan, and \\ ${ }^{5}$ Anatomic Pathology Section, Fondazione IRCCS Policlinico San Matteo, Pavia, Italy
}

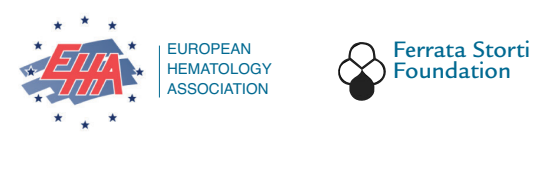

Haematologica 2017

Volume 102(12):2077-2085

\section{ABSTRACT}

W e analyzed MYD88 and CXCR4 mutation status of 260 patients with Waldenström macroglobulinemia or IgM monoclonal gammopathy of undetermined significance using allelespecific real time quantitative polymerase chain reaction and Sanger sequencing, respectively. A subgroup of 119 patients was further studied with next-generation sequencing of 11 target genes (MYD88, CXCR4, ARID1A, KMT2D, NOTCH2, TP53, PRDM1, CD79B, TRAF3, MYBBP1A, and TNFAIP3). MYD88 (L265P) was found at diagnosis in $91 \%$ of patients with Waldenström macroglobulinemia and in $60 \%$ of patients with IgM monoclonal gammopathy of undetermined significance using allele-specific polymerase chain reaction analysis. MYD88 mutations other than the classical L265P (V217F, S219C and M232T) were found in four cases by next-generation sequencing. Waldenström macroglobulinemia patients with wild-type MYD 88 had a distinct clinical phenotype characterized by less bone marrow infiltration $(P=0.01)$ and more frequent extramedullary involvement $(P=0.001)$ compared to patients with mutated MYD88. Patients with wild-type MYD 88 did not show additional mutations in the other target genes. CXCR4 mutations were found by Sanger sequencing in $22 \%$ of patients with Waldenström macroglobulinemia. With next-generation sequencing, a CXCR4 mutation was detected in $23 \%$ of patients with Waldenström macroglobulinemia and $9 \%$ of those with IgM monoclonal gammopathy of undetermined significance. Asymptomatic Waldenström macroglobulinemia patients harboring a CXCR4 mutation had a shorter treatment-free survival (51 months) than that of patients with wild-type CXCR4 (median not reached) $(P=0.007)$. Analysis of variant allele frequencies indicated that CXCR4 mutations were present in the dominant clone in the majority of cases. Recurrent somatic mutations of KMT2D were found in $24 \%$ of patients with Waldenström macroglobulinemia and $5 \%$ of patients with IgM monoclonal gammopathy of undetermined significance and were primarily subclonal.

\section{Introduction}

Waldenström macroglobulinemia (WM) is a rare lymphoproliferative disorder characterized by the presence of a serum IgM paraprotein associated with infiltration of the bone marrow by lymphoplasmacytic lymphoma. ${ }^{1}$ Familial aggregation of WM and related B-cell disorders strongly supports a role for genetic factors in the pathogenesis of the disease..$^{2 \cdot 4}$

\section{Correspondence: \\ m.varettoni@smatteo.pv.it}

Received: May 15, 2017.

Accepted: September 28, 2017

Pre-published: October 5, 2017.

doi:10.3324/haematol.2017.172718

Check the online version for the most updated information on this article, online supplements, and information on authorship \& disclosures: www.haematologica.org/content/102/12/2077

(C)2017 Ferrata Storti Foundation

Material published in Haematologica is covered by copyright. All rights are reserved to the Ferrata Storti Foundation. Use of published material is allowed under the following terms and conditions: Copies of published material are allowed for personal or internal use. Sharing published material for non-commercial purposes is subject to the following conditions: https://creativecommons. org//icenses/by-nc/4.0/legalcode, sect. 3. Reproducing and sharing published material for commercial purposes is not allowed without permission in writing https://creativecommons.org/licenses/by-nc/4.0/legalcode. from the publisher. 
Some years ago, using whole genome sequencing Treon et al. identified MYD88 (L265P) as the most common somatic mutation in WM. ${ }^{5}$ Signaling studies showed that the mutant protein encoded by MYD88 triggers tumor growth through the activation of nuclear factor kappa light-chain enhancer of activated B cells (NF- $\kappa B$ ) via two independent pathways, namely IRAK1-4 and BTK. ${ }^{6}$ The frequent recurrence of one single variant (i.e. L265P) in WM/lymphoplasmacytic lymphoma prompted the design of allele-specific polymerase chain reaction (PCR)-based strategies for the detection of the MYD88 mutation. ${ }^{7-8}$ Using this approach, the MYD88 (L265P) mutation is detectable in more than $90 \%$ of WM patients, while it is rarely expressed in other indolent mature B-cell tumors, such as splenic marginal zone lymphoma or chronic lymphocytic leukemia. ${ }^{7-10}$

The diagnosis of WM may be preceded by a history of IgM monoclonal gammopathy of undetermined significance (IgM MGUS). Long-term follow-up studies show that patients with IgM MGUS have a risk of progression to WM or to other B-cell lymphoproliferative disorders of approximately $1.5-2 \%$ per year. ${ }^{11}$ Using allele-specific PCR, $50-80 \%$ of patients with IgM MGUS were found to harbor the MYD88 (L265P) mutation, suggesting that MYD88 mutation is an early genetic event in the development of WM. ${ }^{7-10,12}$ In a previous study, we demonstrated that IgM MGUS patients harboring the MYD88 (L265P) mutation have a significantly higher risk of progression to WM or other lymphoproliferative disorder as compared with patients with the wild-type gene, regardless of the size of the serum IgM monoclonal protein. ${ }^{13}$

The second most common mutations in WM are nonsense or frameshift mutations in the carboxyl-terminal cytoplasmic tail of the CXCR4 gene. CXCR4 is overexpressed by cancer cells in many hematopoietic and solid cancers, but WM is the first human cancer in which somatic CXCR4 mutations have been reported. ${ }^{14}$ These mutations are similar to germline mutations typical of the WHIM syndrome, an inherited autosomal dominant disorder characterized by warts, hypogammaglobulinemia, infections and myelokathexis. ${ }^{15}$ In WM, somatic CXCR4 mutations result in impaired internalization of the CXCR4 receptor, leading to constitutive activation of the CXCR4 pathway, AKT and ERK activation and eventually WM cell survival. ${ }^{16-17}$ Using Sanger sequencing, CXCR4 mutations are detected in approximately $30 \%$ of WM patients, almost all of whom also harbor the MYD88 (L265P) mutation. CXCR4 mutations are usually subclonal, supporting the notion that they are acquired after the MYD88 (L265P) mutation in the development of WM. ${ }^{18}$ From a clinical standpoint, the presence of CXCR4 mutations has been associated with a more aggressive clinical presentation, including higher levels of IgM serum monoclonal protein, a higher incidence of hyperviscosity syndrome and more extensive bone marrow infiltration, ${ }^{19-21}$ as well as with clinical resistance to Ibrutinib. ${ }^{16-17,22}$

While there is an increasing body of data about MYD 88 and CXCR4 mutations, little is known about the remaining genomic landscape of WM. Using whole genome sequencing, somatic mutations of ARID1A have been found in $17 \%$ of patients, while CD79B, KMT2D (formerly known as MLL2), MYBBP1A and TP53 mutations have been described in less than $10 \%$ of cases each.

The aim of this study was to analyze the pattern of mutations of 11 genes in a cohort of well-annotated WM or IgM MGUS patients, and to evaluate correlations between somatic mutations and disease phenotype or patients' outcome. The following genes were studied: MYD88, CXCR4, ARID1A, KMT2D, NOTCH2, TP53, PRDM1, CD79B, TRAF3, MYBBP1A, and TNFAIP3.

In this study we demonstrated that MYD 88 wild-type patients have a distinct clinical phenotype and do not show additional somatic mutations in the other target genes; furthermore, we demonstrated that CXCR4 mutations in WM patients are associated with an earlier need for treatment. Finally we report for the first time that subclonal KMT2D mutations are highly recurrent in WM patients.

\section{Methods}

These investigations were approved by the local Ethics Committee. The procedures followed were in accordance with the Helsinki Declaration of 1975, as revised in 2000, and samples were obtained after subjects had provided informed consent.

\section{Patients}

The study population included 130 patients with WM and 130 patients with IgM MGUS, in whom MYD88 and CXCR4 mutation status was evaluated with allele-specific real-time quantitative PCR (RT-qPCR) and Sanger sequencing, respectively. The methods are described in the Online Supplements. A subgroup of 119 patients (62 with WM and 57 with IgM MGUS) was further studied using next-generation sequencing (NGS) of 11 target genes, selected on the basis of data available in the literature. The patients' characteristics are reported in Online Supplementary Table S1. The diagnoses of WM and IgM MGUS were made according to the consensus recommendations from the Second International Workshop on WM. ${ }^{1}$

\section{Sample collection and cell separation}

Sample collection and cell separation are described in the Online Supplement.

\section{Mutation analysis of target genes using next-generation sequencing}

Mutation analysis was performed on paired tumor/germline gDNA in order to identify relevant somatic mutations of selected genes. A TruSeq Custom Amplicon panel (TSCA; Illumina, San Diego, CA, USA) targeting complete coding exons and their adjacent splice junctions from the 11 genes was designed using Illumina Design Studio software. The TSCA panel consisted of 249 amplicons, $425 \mathrm{bp}$ in length, for a total of $69 \mathrm{~kb}$ targeted DNA. Dual-barcoded TSCA libraries were created from $250 \mathrm{ng}$ of high quality DNA according to the manufacturer's protocol. Libraries were multiplexed and underwent $2 \times 250-b p$ paired-end sequencing on a MiSeq sequencing system using the MiSeq Reagent Kit version 3 (Illumina). The resulting average depth of coverage for the 249 amplicons was $1009 x$.

Sequence reads were initially aligned to the human genome (GRCh37/hg19) using the Burrows-Wheeler ${ }^{23}$ aligner. The Genome Analysis Toolkit ${ }^{24}$ (www.broadinstitute.org/gatk/) was later used to clean up reads and make alignment data more reliable for the variant calling (GATK data cleanup best practice): single nucleotide variants and small insertions-deletions were identified by Mutect $^{25}$ and UnifiedGenotyper, respectively. Functionally annotated variants were filtered according to the following criteria: synonymous variants and variants located outside protein coding regions were filtered; polymorphisms described in dbSNP (ver- 
sion 138) and the 1000 Genomes Project with a population frequency higher than $1 \%$ and $0.14 \%$, respectively, were removed; variants with coverage $<30 \mathrm{X}$ and less than ten supporting reads and variants with an allelic fraction lower than $1 \%$ were filtered; the remaining variants, evaluated as candidate somatic mutations, were manually reviewed and tagged as oncogenic using different criteria based on information retrieved from the literature, sequence conservation and in silico prediction of effect.

\section{Statistics}

Quantitative variables are summarized in terms of median and range. Categorical variables are described by absolute and relative frequencies. The association between two categorical variables was estimated by the Fisher exact test. Quantitative variables were compared between two groups of patients using the Wilcoxon rank-sum test. The correlation between two quantitative variables was tested using the Spearman rho correlation.

Overall survival was defined as the time from diagnosis to death from any cause or last follow-up (for censored patients) Treatment-free survival was measured (only in WM patients with mutational status evaluated at diagnosis) from the date of diagnosis to the date of first-line treatment or the last follow-up (for censored patients). Overall survival and treatment-free survival were estimated using the Kaplan-Meier product limit method and survival curves were compared by the log-rank test. The effect of variables on overall survival and treatment-free survival was evaluated using the proportional hazard Cox model. The agreement between NGS and PCR results and between methods was tested by the Cohen kappa coefficient. $P$-values less than 0.05 were considered statistically significant. All analyses were carried out using Stata 12.1 software (2011).

\section{Results}

\section{MYD88 mutation status and allele burden as deter- mined by polymerase chain reaction}

Using allele-specific RT-qPCR, the MYD88 (L265P) mutation was found in 78 of $130(60 \%)$ of patients with IgM MGUS and in 112 of 130 (86\%) of those with WM. In WM, 96/106 untreated patients (91\%) and 16/24 (67\%) of previously treated patients were found to harbor the MYD88 (L265P) mutation. Among 24 previously treated WM patients, seven had wild-type MYD88. Two of them had progressive disease with a bone marrow infiltration of $50 \%$ and $70 \%$. The other five MYD 88 wild-type patients were in very good partial response and did not have detectable bone marrow infiltration at the time of analysis. Among the patients with an MYD88 mutation, the median allele burden was 1.05 (range, 0.1-18.4) in those with IgM MGUS and 15.4 (range, 0.1-96) in those with WM $(P<0.001)$. Patients with symptomatic WM had a higher MYD88 allele burden as compared with asymptomatic ones (28.5 versus 13; $P=0.05$ ) (Figure 1). However, there were overlapping values without a clear cut-off between WM and IgM MGUS patients. The prevalence of MYD88 (L265P) mutation was not significantly higher in CD19+-selected bone marrow samples than in unselected samples either in IgM MGUS patients (62\% versus $58 \%$; $P=0.722)$ or in WM patients ( $87 \%$ versus $85 \% ; P=0.802)$.

\section{Correlations of MYD88 mutation status and MYD88 allele burden with disease phenotype}

Compared with WM patients harboring the MYD88 (L265P) mutation, patients with wild-type MYD88 had significantly less bone marrow infiltration (median 18\% versus $35 \% ; P=0.01$ ) and higher $\beta_{2}$-microglobulin levels (median $3109 \mu \mathrm{g} / \mathrm{L}$ versus $2480 \mu \mathrm{g} / \mathrm{L} ; P=0.03$ ), borderline lower IgM levels (median $536 \mathrm{mg} / \mathrm{dL}$ versus $1170 \mathrm{mg} / \mathrm{dL}$; $P=0.07$ ) and platelet counts (median $217 \times 10^{\circ} / \mathrm{L}$ versus 260 $\left.x \quad 10^{9} / \mathrm{L} ; P=0.06\right)$, and more frequent extramedullary involvement $(60 \%$ versus $12 \%$ of patients; $P=0.001)$. No significant difference was found for age, sex or the other clinical characteristics. Among WM patients with the MYD88 (L265P) mutation, the median MYD88 allele burden was significantly higher in patients with hemoglobin levels $<10 \mathrm{~g} / \mathrm{dL}(P=0.01), \beta_{2}$-microglobulin levels $>3000$ $\mu \mathrm{g} / \mathrm{L}(P<0.001)$, serum albumin levels $<3.5 \mathrm{~g} / \mathrm{dL}(P=0.01)$, detectable Bence-Jones proteinuria $(P=0.04)$, abnormal free-light chain ratio $(P=0.046)$ or bone marrow infiltration $>30 \%(P=0.01)$ (Figure 2). No significant correlation was found between MYD88 allele burden and age, sex, platelet count, IgM levels, or presence of extramedullary disease.

\section{CXCR4 mutations in patients with IgM monoclonal gammopathy of undetermined significance and Waldenström macroglobulinemia}

Thirty-four of 260 patients $(13 \%)$ had a CXCR4 mutation. Nonsense and frameshift mutations accounted for 16 $(47 \%)$ and 18 cases (53\%), respectively, C1013G being the most common variant $(14 / 34,41 \%$ of all CXCR4 mutations). The frequency of CXCR4 mutations was 5/130 $(4 \%)$ in IgM MGUS patients and 29/130 (22\%) in WM patients $(P<0.001)$. The prevalence of CXCR4 mutations was not statistically different between $\mathrm{CD} 19^{+}$-selected and unsorted bone marrow samples $7 \%$ versus $2 \%$ in IgM MGUS patients; $P=0.188$ and $25 \%$ versus $19 \%$ in WM patients; $P=0.519$ ). In WM patients, the presence of a CXCR 4 mutation was associated with a significantly higher degree of bone marrow infiltration $50 \%$ versus $30 \%$; $P=0.04)$ and higher MYD 88 allele burden $(24 \%$ versus $9 \%$; $P=0.01$ ) (Online Supplementary Table S2). Taking into account that the threshold for mutant allele detection by Sanger sequencing is approximately $20 \%$, we analyzed the prevalence of CXCR4 mutations according to this cutoff and found that the rate of CXCR 4 mutations was $27 \%$ in patients with $20 \%$ or more bone marrow infiltration

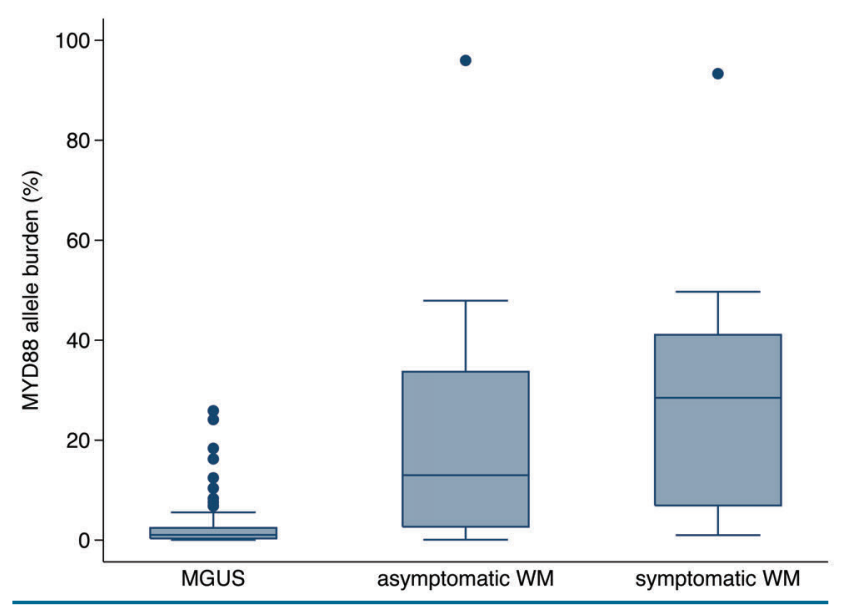

Figure 1. MYD88 allele burden in patients with IgM monoclonal gammopathy of undetermined significance, asymptomatic and symptomatic Waldenström macroglobulinemia. 
and $13 \%$ in patients with less than $20 \%$ bone marrow infiltration $(P=0.179)$.

\section{Prognostic impact of CXCR4 and MYD88 mutations}

The median follow-up of the whole series was 43 months (38.9 months for IgM MGUS patients and 50.4 months for WM patients). During the follow-up, three of 130 IgM MGUS patients (2\%) and 26 of 81 asymptomatic WM patients (32\%) progressed to symptomatic WM requiring treatment. Twenty-five of 260 patients $(10 \%)$ have died.

The median treatment-free survival was significantly shorter in asymptomatic WM patients harboring a CXCR4 mutation at diagnosis (median 51 months) than in those with wild type CXCR4 (median not reached) $(P=0.007)$ (Figure 3). In multivariate analysis, CXCR4 mutation and size of serum protein were independent prognostic factors for progression from asymptomatic to symptomatic WM requiring therapy (Online Supplementary Table S3). MYD88 mutational status did not influence time to first treatment $(P=0.19)$. Treatment-free survival was significantly shorter in asymptomatic WM patients harboring both MYD88 and CXCR4 mutations than in MYD88-mutated/CXCR4wild type patients $(P=0.019)$, confirming that $C X C R 4$ but not MYD88 mutations were associated with an earlier need for treatment (Figure 4). Overall survival was not affected by either MYD 88 or CXCR4 mutational status (data not shown).

\section{Somatic mutations identified by next-generation sequencing}

Overall, 88 of 119 patients studied with NGS (74\%) had one or more somatic mutations in the 11 target genes. The median number of mutations was significantly higher in WM patients than in IgM MGUS patients (median 2 versus 1; $P<0.001)$ and in those previously treated than in untreated ones (median 2 versus 1; $P<0.001$ ). MYD88 mutations were found in $80 / 119$ patients $(67 \%)$, with a median variant allele frequency of $34.2 \%$ (range, 3.0$93.3 \%$ ). The prevalence of MYD 88 mutations determined by NGS was $85 \%$ in WM and $47 \%$ in IgM MGUS patients $(P<0.001)$ (Figure 5). MYD88 mutations other than the classical L265P $(n=76)$ were found in four patients and were V217F $(n=2)$, S219C $(n=1)$ and M232T $(n=1)$. Fourteen patients who resulted MYD88 (L265P) wild-type by NGS were found to have the mutation by allele-specific RT-qPCR (K coefficient of concordance between NGS and PCR, $65 \% ; P<0.001)$. Twelve of them had a diagnosis of IgM MGUS and two had WM. Of these latter two patients, one was untreated with predominant extramedullary disease and minimal bone marrow infiltration, the other was a previously treated patient who had achieved a very good partial response and did not have detectable bone marrow infiltration at the time of mutation status analysis.

Using NGS a CXCR4 mutation was found in 19 patients $(16 \%)$ with a median variant allele frequency of $22.5 \%$ (range, 4.2-49.8\%). The $\mathrm{K}$ coefficient of concordance between NGS and Sanger sequencing was $89.8 \%$ $(P<0.001)$. The prevalence of CXCR 4 mutations was $23 \%$ in WM and $9 \%$ in IgM MGUS patients $(P=0.047)$ (Figure 5). Of the two patients with a $C X C R 4$ mutation who were MYD88-wild type according to NGS, one was found to harbor the MYD88 (L265P) mutation with a low allele burden $(0.34 \%)$ using allele-specific RT-qPCR. The comparison of variant allele frequencies of CXCR4 and MYD88 mutations in individual patients indicated that CXCR4 mutations were present in the dominant clone in the majority of cases (Figure 6).

NGS allowed the identification of somatic mutations in KMT2D (16\% of patients), TP53 (8\%), NOTCH2 (7\%), PRDM1 (4\%), ARID1A (3\%), CD79B (3\%), and TRAF3
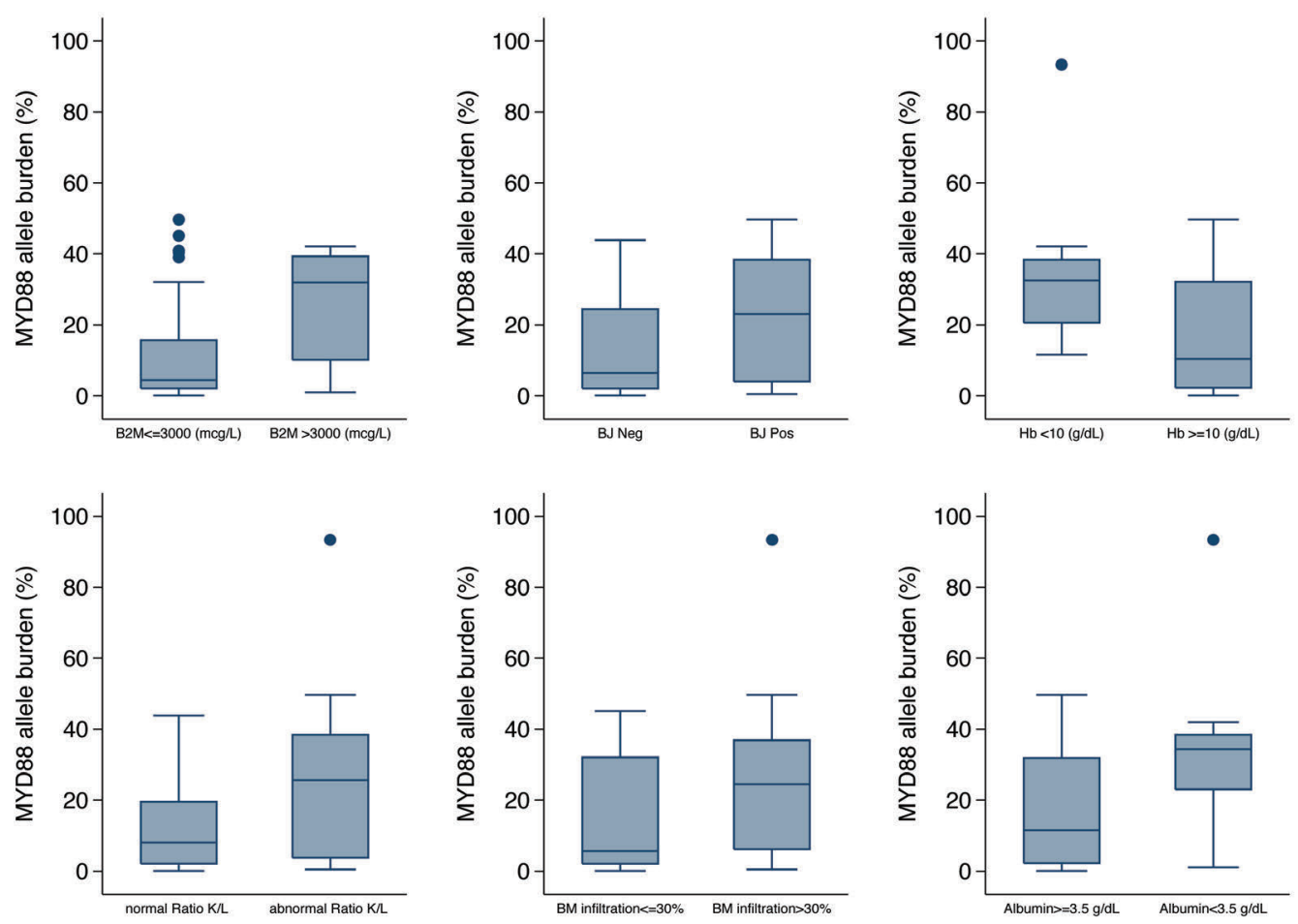

Figure 2. Correlation of MYD88 allele burden with disease phenotype in patients with Waldenström macroglobulinemia. 
(1\%). No mutations were found in MYBBP1A or TNFAIP3. Overall, the prevalence of these mutations was significantly lower in patients with either wild-type MYD88 or wild-type CXCR4 than in those with MYD88 and/or CXCR4 mutations (16\% versus $41 \% ; P=0.007$ ) (Figure 5 ). The prevalence of KMT2D mutations was significantly higher in WM patients than in patients with IgM MGUS ( $24 \%$ versus $5 \%$; $P=0.002$ ) while the prevalence of mutations in other genes was not statistically different according to diagnosis ( $P>0.3$ for all comparisons). The analysis of variant allele frequency in patients harboring either a MYD88 or a KMT2D mutation showed that KMT2D mutations were primarily subclonal (Figure 7).

TP53 mutations were found in three of 57 patients with IgM MGUS (5\%) and six of 62 patients with WM (10\%). The variant allele frequency of TP53 mutations was below $3.5 \%$ in IgM MGUS patients and untreated WM patients. In the four previously treated WM patients, the variant allele frequencies were $2.9 \%, 10.1 \%, 11.2 \%$ and $57.8 \%$.
We did not find a statistically significant correlation of KMT2D, TP53, NOTCH2, PRDM1, ARID1A, CD79B, or TRAF3 mutations with overall survival or time to first treatment.

\section{Discussion}

This study was conducted on two well-balanced subgroups of patients with an established diagnosis of IgM MGUS or WM. The study includes the largest series published so far of IgM MGUS patients screened for somatic mutations of genes with potential relevance to the pathogenesis of WM. Since progression from IgM MGUS to WM is likely a multi-step process, in which multiple genetic hits are required for progression from a pre-benign condition to an overt neoplastic disease, the analysis of mutation pattern in IgM MGUS patients is useful to understand whether a given mutation represents an early
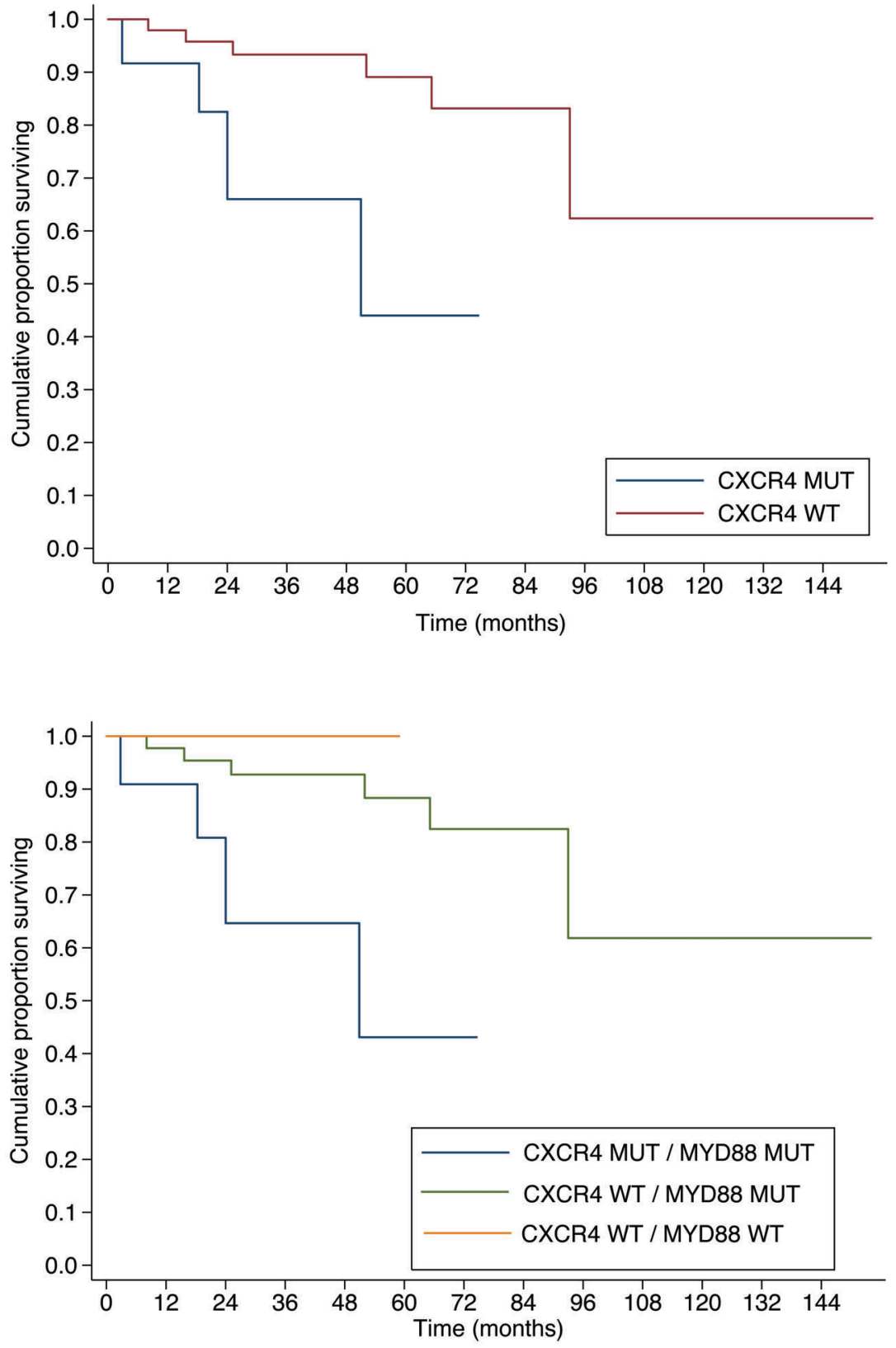

Figure 3. Treatment-free survival of asymptomatic patients with Waldenström macroglobulinemia according to CXCR4 mutation status. 
or late event during the oncogenic process starting from MGUS and ending with WM. In agreement with this model, the high prevalence of MYD88 mutations in IgM MGUS patients $(60 \%)$ confirms that this mutation is an early event, while the low prevalence of CXCR4 and KMT2D mutations in IgM MGUS (<10\%) suggests that these mutations usually occur later.
As expected, the MYD88 (L265P) mutation was confirmed as the most common somatic mutation in WM and IgM MGUS, in agreement with results reported by other groups. ${ }^{5,-10,12-14}$ Among the patients with WM, the rate of MYD88 (L265P) mutation was significantly lower in previously treated patients than in untreated ones. The majority of WM patients who resulted MYD 88 wild-type
A

\begin{tabular}{|c|c|}
\hline MYD88 & 47\% |||||||||||||||||||||||||||||||||||||||||||||||||||| \\
\hline Кмт2D & 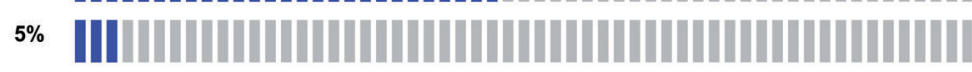 \\
\hline CXCR4 & 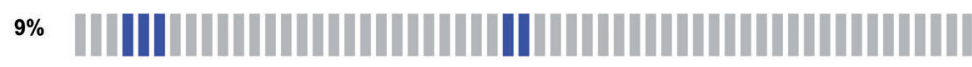 \\
\hline TP53 & 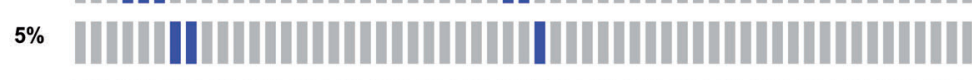 \\
\hline PRDM1 & 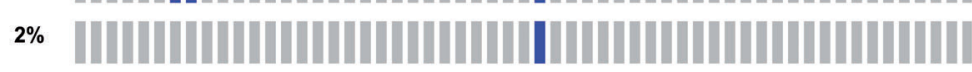 \\
\hline CD79B & 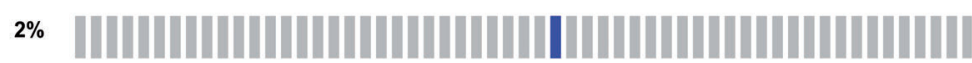 \\
\hline ARID1A & 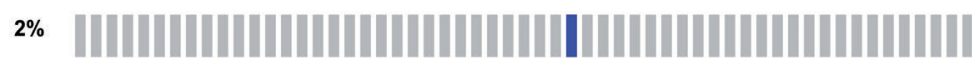 \\
\hline & 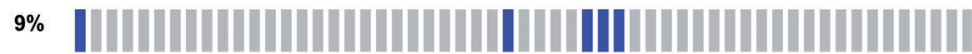 \\
\hline
\end{tabular}

B
Figure 5. Pattern of mutations observed in patients with (A) IgM monoclonal gammopathy of undetermined significance or (B) Waldenström macroglobulinemia.

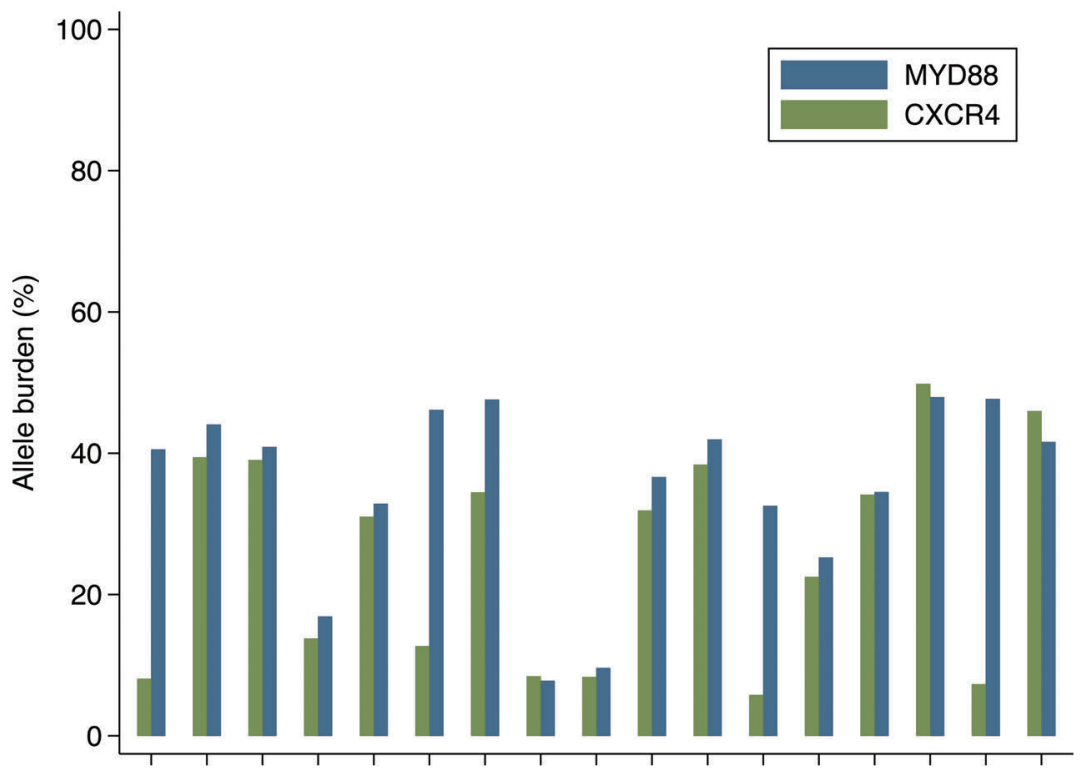

Figure 6. Variant allele frequency of MYD88 and CXCR4 mutations as determined by next-generation sequencing. 
after therapy had been successfully treated with immunochemotherapy. The lower yield of clonal B cells following treatment with B-cell depleting agents may at least in part explain this finding, even though a baseline sample was not available to confirm this hypothesis.

Samples studied with RT-qPCR for MYD88 (L265P) included either $\mathrm{CD} 19^{+}$-selected or unselected bone marrow mononuclear cells. The rate of MYD88 (L265P) mutation was not significantly higher in sorted samples than in unsorted ones, either in IgM MGUS or in WM. This result was not surprising in WM patients, in line with a recent publication showing a high level of concordance in the rate of MYD88 (L265P) mutation between selected and unselected bone marrow cells, ${ }^{26}$ while it was less expected in the context of IgM MGUS. In $\mathrm{CD}_{19}{ }^{+}$-selected samples, we did not assess CD19+ clonality with light chain restriction, which would probably have increased the accuracy in detecting the MYD88 (L265P) mutation, especially among patients with IgM MGUS. In fact, using this approach the Salamanca group found the MYD88 (L265P) mutation in $87 \%$ of IgM MGUS patients, which is a higher rate than in our study as well as in other published series. ${ }^{9}$ Finally, we did not specifically evaluate the plasma cell compartment, in which the presence of the MYD88 (L265P) mutation has been previously reported. 5,26 These observations indicate that the source of DNA may affect the rate of MYD 88 mutations, and underline the need to define the best strategy for testing these mutations, balancing the gain in accuracy with time and costs required for $\mathrm{CD} 19^{+}$sorting and light chain restriction assessment.

NGS allowed the identification of mutations other than the classical L265P variant in four patients. Among these, the M232T variant has been recently described in a WM patient, ${ }^{27}$ whereas the V217F and S219C had been previously reported in diffuse large B-cell lymphoma, activated B-cell subtype..$^{28}$ Overall, there was a strong concordance between NGS and RT-qPCR findings, and discordant cases were mainly represented by IgM MGUS patients in whom the L265P mutation was found only with RTqPCR. This finding confirms the greater sensitivity of RT-
qPCR over NGS, which becomes evident when the B-cell clone is small.

WM patients with wild-type MYD 88 showed distinct clinical features, including lower IgM levels, less bone marrow infiltration and more frequent extramedullary disease as compared with patients harboring the MYD 88 mutation. MYD 88 wild-type patients were almost invariably CXCR4 wild-type and did not have additional mutations in the other genes studied with NGS. The existence of a small subgroup of WM patients not harboring the MYD88 (L265P) mutation has been increasingly recognized in recent studies. These patients seem to have lower response rates to the BTK inhibitor ibrutinib and a poorer outcome as compared with MYD88-mutated patients. ${ }^{19,22}$ Furthermore, MYD 88 wild-type cases show lower expression of genes related to B-cell differentiation and a lower rate of IGH somatic hypermutation, suggesting that they constitute a distinct entity with respect to classical WM possibly deriving from a $B$ cell in an earlier stage of differentiation. ${ }^{9,29}$

Allele-specific RT-qPCR allowed the estimation of MYD88 allele burden. Although WM patients usually have higher allele burdens as compared with IgM MGUS patients, we could not identify a threshold that reliably distinguished these two conditions.

In WM patients, the MYD 88 allele burden was found to be a good surrogate marker of clinical disease burden. Although clinical variables associated with a higher MYD88 allele burden include some of the prognostic factors of the WM International Prognostic Scoring System (hemoglobin, albumin, $\beta_{2}$-microglobulin levels), a higher MYD88 allele burden was not associated with worse survival.

In this study we analyzed CXCR4 mutation status by means of Sanger sequencing and NGS. The threshold for mutant allele detection by Sanger sequencing is approximately $20 \%$ and the rate of CXCR 4 mutations was twofold higher in patients with a bone marrow infiltration of $20 \%$ or more than in patients with a bone marrow infiltration of less than $20 \%$. Given the greater sensitivity of

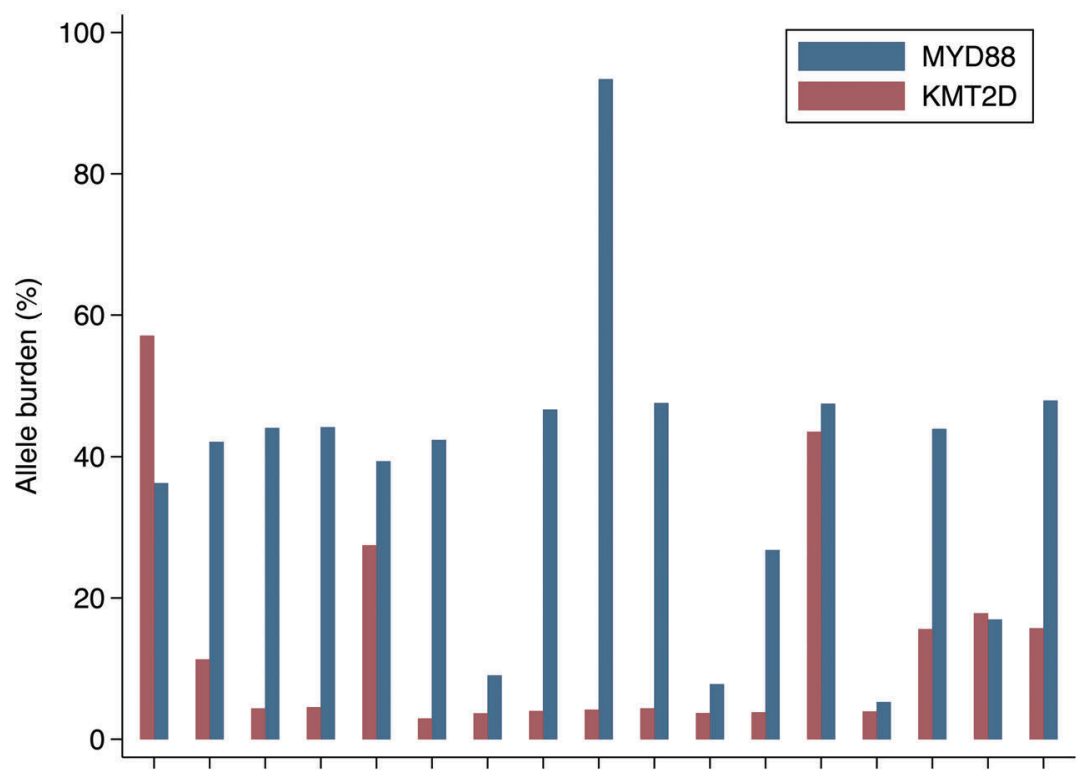

Figure 7. Variant allele frequency of $M Y D 88$ and KMT2D mutations as determined by next-generation sequencing. 
NGS, we expected to find a significantly higher rate of mutations with this technique than with Sanger sequencing, especially in patients with IgM MGUS in whom the $\mathrm{B}$-cell clone is usually under the detection limits of Sanger sequencing. Indeed, less than $10 \%$ of IgM MGUS patients were found to harbor a CXCR4 mutation by NGS, suggesting that CXCR4 mutations are later events than the MYD88 (L265P) mutation in the pathogenesis of WM/lymphoplasmacytic lymphoma. A CXCR 4 mutation was found in approximately a quarter of WM patients, in agreement with results reported by Poulain et al. in a series of WM patients studied with Sanger sequencing and NGS. ${ }^{20}$ Combining Sanger technology with allele-specific PCR for the most common variant S338X, CXCR4 mutations were found in up to $43 \%$ of WM patients, suggesting that differences among studies depend mainly on the sensitivity of the method used as well as the selection of patients. ${ }^{18}$

In a study published by Xu et al., CXCR4 mutations were shown by cancer cell fraction analysis to be primarily subclonal. ${ }^{18}$ Although in a targeted sequencing approach with DNA it is often difficult to determine unequivocally whether a mutation is clonal or subclonal, because of problems with determining the tumor cell content in the biopsy, lack of knowledge about aneuploidy of chromosomes with mutations, and potential variation in the efficiency of amplifying the wild-type and mutated alleles, our data seem to indicate that CXCR4 mutations pertain to the dominant MYD88-mutated clone in the majority of cases.

Of interest, CXCR4 mutations were associated with an earlier need for treatment in patients with WM. In a study of 48 asymptomatic WM patients with a long follow-up, the cumulative probability of progression to symptomatic WM or other lymphoproliferative disorders was $59 \%$ at 5 years and $68 \%$ at 10 years. The main risk factors for progression were the extent of bone marrow infiltration by lymphoplasmacytic cells, the size of monoclonal protein and hemoglobin levels. ${ }^{30}$ In our study we performed a multivariate analysis including CXCR4 mutation status and the above reported clinical risk factors for progression. CXCR4 mutation status and the size of serum monoclonal protein were independent risk factors for progression from asymptomatic to symptomatic WM requiring therapy. Based on these findings, a model including molecular and clinical variables may refine prognostication of asymptomatic WM patients and potentially lead to the design of risk-adapted follow-up strategies.

In the subgroup of patients studied with NGS we analyzed the occurrence of somatic mutations in other genes with potential relevance in WM based on evidence from the literature. One of the main strengths of this study is that we could validate the somatic origin of the mutations as a matched control, represented by bone marrow CD19mononuclear cells was available in all cases. We found that subclonal mutations of KMT2D are highly recurrent in WM patients and may also be present in IgM MGUS patients. KMT2D encodes a histone methyltransferase that targets the Lys-4 position of histone H3. Somatically acquired KMT2D mutations have been reported in about $90 \%$ of follicular lymphomas, $30 \%$ of diffuse large B-cell lymphomas, $15 \%$ of splenic marginal zone lymphomas, as well as in $10-15 \%$ of mantle cell lymphomas. ${ }^{31-35}$ Germline KMT2D mutations have been reported in 60$70 \%$ of patients with Kabuki syndrome, an autosomal dominant disorder characterized by craniofacial, intellectual, and cardiac abnormalities as well as by recurrent infections. In these patients, KMT2D mutations are associated with dysregulation of terminal B-cell differentiation, leading to humoral immune deficiency and autoimmune complications. ${ }^{36}$ Abnormally low serum levels of IgA, IgG, or both have been observed in more than $50 \%$ of WM patients before treatment and remain low after treatment also in patients achieving a complete remission. ${ }^{37}$ Whether there is a relationship between KMT2D mutations and hypogammaglobulinemia or autoimmune phenomena commonly observed in WM patients deserves further investigations.

Using NGS we found TP53 mutations in 10\% of WM patients and in 5\% of IgM MGUS patients; however, so far these mutations have not been seen to have a prognostic impact, probably because of the short follow-up of patients studied with NGS. Interestingly, Poulain et al. recently reported a similar prevalence of TP53 mutations in WM $(11 \%)$ and showed that TP53 alterations were associated with shorter overall survival, independently of WM International Prognostic Scoring System score. ${ }^{38}$

In conclusion, this study adds more knowledge about the clinical and prognostic implications of MYD 88 and CXCR4 mutations and reveals the presence of further somatic mutations potentially relevant to the pathogenesis of WM. Longitudinal studies with sequential evaluations during the course of the disease are needed to understand the clonal evolution underlying progression from IgM MGUS to WM and clonal dynamics under treatment.

\section{Acknowledgments}

This study was supported by Fondazione Cariplo \& Regione Lombardia, Milan (grant ID 42916996) to MGDP.

\section{References}

1. Owen RG, Treon SP, Al-Katib A, et al. Clinicopathological definition of Waldenstrom's macroglobulinemia: consensus panel recommendations from the Second International Workshop on Waldenstrom's Macroglobulinemia. Semin Oncol. 2003;30(2):110-115.

2. Royer RH, Koshiol J, Giambarresi TR, Vasquez LG, Pfeiffer RM, McMaster ML. Differential characteristics of Waldenström macroglobulinemia according to patterns of familial aggregation. Blood. 2010;115(22): 4464-4471.

3. Kristinsson SY, Björkholm M, Goldin LR, McMaster ML, Turesson I, Landgren O. Risk of lymphoproliferative disorders among first- degree relatives of lymphoplasmacytic lymphoma/Waldenstrom macroglobulinemia patients: a population-based study in Sweden. Blood. 2008;112(8):3052-3056.

4. Treon SP, Hunter ZR, Aggarwal A, et al. Characterization of familial Waldenstrom's macroglobulinemia. Ann Oncol. 2006;17(3): 488-494.

5. Treon SP, Xu L, Yang G, et al. MYD88 L265P somatic mutation in Waldenstrom's macroglobulinemia. N Engl J Med. 2012;367(9): 826-833.

6. Yang G, Zhou Y, Liu X, et al. A mutation in MYD88 (L265P) supports the survival of lymphoplasmacytic cells by activation of Bruton tyrosine kinase in Waldenstrom macroglobulinemia. Blood. 2013;122(7): 1222-1232.

7. Varettoni M, Arcaini L, Zibellini S, et al. Prevalence and clinical significance of the MYD88 (L265P) somatic mutation in Waldenstrom's macroglobulinemia and related lymphoid neoplasms. Blood. 
2013;121(13):2522-2528

8. Xu L, Hunter ZR, Yang G, et al. MYD88 L265P in Waldenstrom macroglobulinemia, immunoglobulin $\mathrm{M}$ monoclonal gammopathy, and other B-cell lymphoproliferative disorders using conventional and quantitative allele-specific polymerase chain reaction. Blood. 2013;121(11):2051-2058.

9. Jimenez C, Sebastian E, Chillon MC, et al. MYD88 L265P is a marker highly characteristic of, but not restricted to, Waldenstrom's macroglobulinemia. Leukemia. 2013;27(8): 1722-1728.

10. Poulain S, Roumier C, Decambron A, et al. MYD88 L265P mutation in Waldenstrom macroglobulinemia. Blood. 2013;121(22): 4504-4511.

11. Kyle RA, Therneau TM, Rajkumar SV, et al. Long-term follow-up of IgM monoclonal gammopathy of undetermined significance. Blood. 2003:102(10):3759-3764.

12. Landgren O, Staudt L. MYD88 L265P somatic mutation in IgM MGUS. N Engl J Med. 2012;367(23):2255-2256.

13. Varettoni M, Zibellini S, Arcaini L, et al. MYD88 (L265P) mutation is an independent risk factor for progression in patients with IgM monoclonal gammopathy of undetermined significance. Blood. 2013;122(13): 2284-2285.

14. Hunter ZR, Xu L, Yang G, et al. The genomic landscape of Waldenstrom macroglobulinemia is characterized by highly recurring MYD88 and WHIM-like CXCR4 mutations, and small somatic deletions associated with B-cell lymphomagenesis. Blood. 2014;123 (11):1637-1646.

15. Hernandez PA, Gorlin RJ, Lukens JN, et al. Mutations in the chemokine receptor gene CXCR4 are associated with WHIM syndrome, a combined immunodeficiency disease. Nat Genet. 2003;34(1):70-74

16. Roccaro AM, Sacco A, Jimenez C, et al. C1013G/CXCR4 acts as a driver mutation of tumor progression and modulator of drug resistance in lymphoplasmacytic lymphoma. Blood. 2014;123(26):4120-4131.

17. Cao Y, Hunter ZR, Liu X, et al The WHIMlike CXCR4(S338X) somatic mutation activates AKT and ERK, and promotes resistance to ibrutinib and other agents used in the treatment of Waldenstrom's macroglobulinemia. Leukemia. 2015;29(1): 169-176.

18. Xu L, Hunter ZR, Tsakmaklis N, et al. Clonal architecture of CXCR4 WHIM-like mutations in Waldenström macroglobulinaemia. Br J Haematol. 2016;172(5):735-744.

19. Treon SP, Cao Y, Xu L, Yang G, Liu X, Hunter ZR. Somatic mutations in MYD88 and CXCR4 are determinants of clinical presentation and overall survival in Waldenstrom macroglobulinemia. Blood. 2014;123(18):2791-2796.

20. Poulain S, Roumier C, Venet-Caillault A, et al. Genomic landscape of CXCR4 mutations in Waldenström macroglobulinemia. Clin Cancer Res. 2016:22(6):1480-1488.

21. Schmidt J, Federmann B, Schindler N, et al MYD88 L265P and CXCR4 mutations in lymphoplasmacytic lymphoma identify cases with high disease activity. Br J Haematol. 2015;169(6):795-803.

22. Treon SP, Tripsas CK, Meid K, et al. Ibrutinib in previously treated Waldenström's macroglobulinemia. N Engl J Med. 2015;372(15):1430-1440.

23. Li H, Durbin R. Fast and accurate long-read alignment with Burrows-Wheeler transform. Bioinformatics. 2010;26(5):589-595.

24. DePristo MA, Banks E, Poplin R, et al. A framework for variation discovery and genotyping using next-generation DNA sequencing data. Nat Genet. 2011;43(5):491498.

25. Cibulskis K, Lawrence MS, Carter SL, et al. Sensitive detection of somatic point mutations in impure and heterogeneous cancer samples. Nat Biotechnol. 2013;31(3):213219.

26. Gustine J, Meid K, Xu L, Hunter ZR, Castillo IJ, Treon SP. To select or not to select? The role of B-cell selection in determining the MYD88 mutation status in Waldenström macroglobulinaemia. $\mathrm{Br} J$ Haematol. 2017;176(5):822-824

27. Treon SP, Xu L, Hunter Z. MYD88 Mutations and response to ibrutinib in Waldenström's macroglobulinemia. N Engl J Med. 2015;373(6):584-586.
28. Ngo VN, Young RM, Schmitz R, et al Oncogenically active MYD88 mutations in human lymphoma. Nature. 2011;470(7332): 115-119.

29. Hunter ZR, Xu L, Yang G, et al. Transcriptome sequencing reveals a profile that corresponds to genomic variants in Waldenström macroglobulinemia. Blood. 2016;128(6):827-838

30. Kyle RA, Benson JT, Larson DR, et al Progression in smoldering Waldenstrom macroglobulinemia: long-term results. Blood. 2012;119(19):4462-4466.

31. Beà $S$, Valdés-Mas R, Navarro A, et al Landscape of somatic mutations and clonal evolution in mantle cell lymphoma. Proc Natl Acad Sci USA. 2013;110(45):1825018255.

32. Morin RD, Mendez-Lago M, Mungall AJ, et al. Frequent mutation of histone-modifying genes in non-Hodgkin lymphoma. Nature. 2011:476(7360):298-303.

33. Okosun J, Bodor C, Wang J, et al. Integrated genomic analysis identifies recurrent mutations and evolution patterns driving the initiation and progression of follicular lymphoma. Nat Genet. 2014;46(2):176-181.

34. Rossi D, Trifonov V, Fangazio M, et al. The coding genome of splenic marginal zone lymphoma: activation of $\mathrm{NOTCH} 2$ and other pathways regulating marginal zone development. J Exp Med. 2012;209(9):15371551.

35. Zhang J, Jima $D$, Moffitt $A B$, et al. The genomic landscape of mantle cell lymphoma is related to the epigenetically determined chromatin state of normal B cells. Blood. 2014;123(19):2988-2996.

36. Lindsley AW, Saal HM, Burrow TA, et al Defects of B-cell terminal differentiation in patients with type-1 Kabuki syndrome. Allergy Clin Immunol. 2016;137(1):179-187.

37. Hunter ZR, Manning RJ, Hanzis C, et al. IgA and $\operatorname{IgG}$ hypogammaglobulinemia in Waldenström's macroglobulinemia. Haematologica. 2010;95(3):470-475.

38. Poulain S, Roumier C, Bertrand E, et al. TP53 mutation and its prognostic significance in Waldenstrom's macroglobulinemia. Clin Cancer Res. 2017 Jul 28. [Epub ahead of print] 